\title{
Preventive Effect of Cryotherapy on Persistent Chemotherapy-induced Peripheral Neuropathy After Completion of Weekly Paclitaxel Therapy for Breast Cancer
}

Hideo Shigematsu ( $\nabla$ shigematu1330@yahoo.co.jp )

kure medical center and chugoku cancer center https://orcid.org/0000-0001-9393-9655

\section{Yuri Kimura}

kure medical center and chugoku cancer center

Tomoko Itagaki

Kure medical center and Chugoku Cancer Center

Daisuke Yasui

Kure medical center and Chugoku cancer center

\section{Research Article}

Keywords: breast cancer, persistent CIPN, cryotherapy, weekly paclitaxelv

Posted Date: May 11th, 2021

DOI: https://doi.org/10.21203/rs.3.rs-417227/v1

License: (c) (1) This work is licensed under a Creative Commons Attribution 4.0 International License.

Read Full License 


\section{Abstract}

\section{Background}

Chemotherapy-induced peripheral neuropathy (CIPN) is an important adverse event of taxane-based chemotherapy, which can persist in a substantial proportion of patients for years. Cryotherapy therapy is shown to be effective in prevention of CIPN during chemotherapy, but its protective effect on persistent CIPN has not been reported.

\section{Methods}

Cases enrolled in a randomized trial investigating the preventive effect of cryotherapy on weekly paclitaxel-induced CIPN in breast cancer patients (UMIN000034966) were evaluated for Functional Assessment of Cancer Therapy-Neurotoxicity (FACT-NTX) score and Patient Neurotoxicity Questionnaire (PNQ) at more than one year after completion of weekly paclitaxel.

\section{Results}

Thirty-eight cases were evaluated for persistent CIPN with a median 2.3 (1.3-3.1) years after completion of weekly paclitaxel. The incidence of a significant decrease in FACT-NTX scores was numerically lower in the cryotherapy group compared with the control group $(15.8 \%$ vs. $36.8 \%, p=0.13)$. There was also a lower grade of PNQ sensory $(p=0.02)$ and motor $(p=0.17)$ in the cryotherapy group compared with the control group.

\section{Conclusion}

In breast cancer patients treated with weekly paclitaxel, cryotherapy resulted in a numerical decrease in the incidence of persistent CIPN at more than one year after completion of weekly paclitaxel treatment.

\section{Introduction}

Chemotherapy-induced peripheral neuropathy (CIPN) is an important adverse event associated with weekly paclitaxel for breast cancer, which interferes with activities of daily living and results in a significant deterioration in quality of life $[5,12]$. Recent studies showed the possibility of a preventive effect with the use of cryotherapy or compression therapy on taxane-related CIPN in breast cancer patients $[4,15,18]$. In a phase II multicenter study, compression therapy using surgical gloves similarly prevented nanoparticle albumin-bound paclitaxel-related CIPN in breast cancer patients. There was a lower incidence of CTCAE grade2 or higher of sensory ( $21.4 \%$ vs. $76.1 \%)$ and motor $(26.2 \%$ vs. $57.1 \%)$ peripheral neuropathies in treated hands than in control hands. In a prospective self-controlled trial evaluating the effect of frozen gloves and socks on weekly paclitaxel related-CIPN, the incidence of objective and subjective CIPN was significantly lower on the intervention side than on the control side at a cumulative paclitaxel dose of $960 \mathrm{mg} / \mathrm{m}^{2}$. A decrease in tactile sensation was recognized in 28 and $25 \%$ of patients in treated hands and feet, respectively, while 81 and $64 \%$ of patients reported having a 
decrease in tactile sensation in the control hand. The Patient Neurotoxicity Questionnaire (PNQ) survey showed a lower incidence of severe neuropathy in the treated side (3\% for hand, $3 \%$ for foot) compared with the control side ( $42 \%$ for hand, $36 \%$ for foot). We also showed a preventive effect of cryotherapy on weekly paclitaxel-related CIPN in a randomized clinical trial (UMIN000034966)[15]. In our study, the proportion of patients with clinically significant CIPN was significantly lower in the cryotherapy group than in the control group ( $41 \mathrm{vs.} 73 \%, p=0.03$ ). Although these studies showed the preventive effect of cryotherapy or compression therapy on CIPN during paclitaxel treatment, these protective effects on CIPN years after completion of paclitaxel treatment have not been evaluated.

To clarify the preventive effect of cryotherapy on taxane-related CIPN after completion of treatment, we surveyed the incidence of CIPN at more than one year after completion of treatment in patients who had been registered in our previous randomized trial.

\section{Methods}

\section{Study design and participants}

Of the 44 cases in a randomized trial investigating the preventive effect of cryotherapy on weekly paclitaxel-induced CIPN in breast cancer patients (UMIN000034966), cases that could provide a response to a questionnaire for CIPN at more than one year after completion of paclitaxel treatment were evaluated in this study. Detailed information and results of the randomized trial are described in a previous report [15]. Briefly, the main inclusion criterion was a diagnosis of invasive breast cancer scheduled for 12 weekly doses of paclitaxel-based treatment. HER2 targeting therapy was combined with weekly paclitaxel in HER2-positive breast cancer. Eligible cases were randomly (1:1) assigned to the cryotherapy group or control group. As cryotherapy, patients continuously wore frozen $\left(-20^{\circ} \mathrm{C}\right)$ gloves/socks (Elasto-Gel, mittens, and slippers) on both hands and feet between $15 \mathrm{~min}$ before and after paclitaxel infusion (total $90 \mathrm{~min}$ ). The primary endpoint was incidence of clinical meaningful CIPN after completion of weekly paclitaxel. In total, 38 cases were given a questionnaire for CIPN between April 2020 and October 2020. Six cases were excluded from this survey due to the following reasons: systemic treatment for recurrent breast cancer (2), transfer to different hospital (2), depression (1), and severe neuropathy after meningioma surgery (1) (Fig. 1). This study was approved by the Ethics Committee of the National Hospital Organization of Kure Medical Center and Chugoku Cancer Center (Approval number 28-70) and adhered to the Helsinki Declaration and the ethical principles for clinical research. All patients received a detailed explanation of the study from the primary physician and informed consent was obtained prior to enrollment.

\section{Evaluation of CIPN}

Eligible cases were evaluated using a questionnaire survey for Functional Assessment of Cancer Therapy-Neurotoxicity (FACT-NTX) score and Patient Neurotoxicity Questionnaire. FACT-NTX consists of 11 items evaluating CIPN-associated symptoms with a 0 to 4 score and a lower FACT-NTX score reflects worse peripheral neuropathy[3]. A 6-point or more decrease in the total FACT-NTX score from baseline is 
regarded as clinically significant CIPN. PNQ is a patient-reported questionnaire on sensory and motor neuropathy and how they influence daily activity and quality of life. A PNQ grade ranges from A (no symptoms) to $E$ (severe symptom with significant deterioration of daily activity)[16].

\section{Statistical analysis}

Patient characteristics were assessed by groups using a Fisher's exact test of categorical variables and ttest for continuous variables. A Fisher's exact test was used to compare the incidence of a clinically significant decrease of the FACT-NTX score and the distributions of each FACT-NTX item and PNQ grade between the cryotherapy and control groups. Predictive factors for a clinically significant decrease of the FACT-NTX score were evaluated using logistic regression analysis. A two-sided $p$ value $<0.05$ was considered significant. Statistical analyses were performed using JMP software version 13.2.1 (SAS Institute Inc., Cary, USA).

\section{Findings}

\section{Patient Characteristics}

Of 38 cases evaluated for persistent CIPN at more than one year after completion of treatment, 19 cases were from the cryotherapy group and 19 were from the control group. The median time from completion of the weekly paclitaxel treatment to questionnaire for persistent CIPN in this study was 2.3 (1.3-3.1) years. There was no statistical difference in regard to baseline clinicopathological factors, including interval from completion of paclitaxel, PS, age, height, weight, stage, subtype, adjuvant endocrine therapy and radiation therapy between the cryotherapy and control group (Table 1). During weekly paclitaxel treatment, there was significantly lower incidence of a clinically significant decrease in FACT-NTX score $(31.6 \%$ vs. $73.7 \%, p=0.008)$ and a lower grade of PNQ sensory $(p=0.02)$ and motor $(p=0.04)$ in the cryotherapy group compared to the control group.

\section{Effect of cryotherapy on incidence of persistent CIPN}

All 38 cases responded to the questionnaire for FACT-NTX and PNQ after completion of weekly paclitaxel treatment. The incidence of a clinically significant decrease in the FACT-NTX score was $15.8 \%$ and $36.8 \%$ in the cryotherapy and control group, respectively, showing a numerically lower incidence in the cryotherapy group $(p=0.14)$ (Table 2$)$. Figure 1 shows the distribution of scores for each questionnaire item in FACT-NTX. There was tendency for a lower score for Hand numbness, Foot numbness and Foot discomfort in the cryotherapy group compared with the control group. In the questionnaire for PNQ, there was also a lower grade of sensory $(p=0.02)$ and motor $(p=0.17)$ symptoms in the cryotherapy group compared with the control group (Table 2).

\section{Predictive factor for persistent CIPN}


Logistic regression analysis was used to evaluate the association between clinicopathological factors and the incidence of significant decrease in the FACT-NTX score. Age 60 years or older had a significantly increased risk of significant decrease in the FACT-NTX score (odds ratio $=26.6,95 \% \mathrm{Cl}=3.3-574.5, \mathrm{p}=$ 0.001). Cryotherapy was marginally associated with significant decrease in the FACT-NTX score (odds ratio $=4.895 \% \mathrm{Cl}=0.7-50.1, \mathrm{p}=0.12$ ). The other baseline clinicopathological factors including age, cryotherapy height, weight, adjuvant endocrine therapy, adjuvant radiation therapy and length of interval, were not associated with significant decrease in the FACT-NTX score.

\section{Discussion}

In this follow-up study of a randomized trial investigating the preventive effect of cryotherapy on weekly paclitaxel-induced CIPN of breast cancer patients, cryotherapy resulted in a numerical decrease in the incidence of persistent CIPN at more than one year after completion of treatment. In this study, logistic regression analysis showed age 60 years or older was a significant predictive factor for persistent CIPN.

CIPN is a common and prolonged adverse event of weekly paclitaxel which has a negative impact on quality of life[5]. With the introduction of target treatment for breast cancer, a substantial proportion of patients have excellent prognosis after adjuvant weekly paclitaxel-containing therapy. For example, adjuvant weekly paclitaxel and trastuzumab therapy resulted in a 7-year OS of $95 \%$ and 7-year RFI of 97.5\% in HER2-positive and node negative breast cancer[17]. Thus, long-term CIPN has become an important issue for the growing number of breast cancer survivors who received adjuvant weekly paclitaxel. Although the degree of paclitaxel-induced CIPN reduces over time, a substantial proportion of the population continues to suffer from CIPN even years after completion of taxane-based chemotherapy[13]. A cross-sectional study of 50 consecutive early-stage breast cancer patients who were within 6 and 24 months of completing adjuvant paclitaxel therapy showed that $80 \%$ of the patients reported numbness or discomfort in the hands or feet and a quarter of the population suffered from severe symptoms[8]. A prospective study of 50 patients also showed that two-thirds of the population reported persistent numbness in the hands or feet, including a quarter with severe symptoms at 12 months after completion of paclitaxel treatment. A randomized study, which evaluated the preventive effect of Acetyl-L-Carnitine on taxane-related CIPN, showed prolonged clinically significant CIPN in more than 30 percent of the population at week 12, 24, 36, 52, 104 after registration[7]. These results show that taxane-related CIPN can persist over a year and persistent CIPN is an important clinical issue to be resolved in breast cancer patients treated with taxane-based chemotherapy. Our study also showed that a substantial proportion of cases have persistent CIPN after completion of weekly paclitaxel. Persistent CIPN was observed in 10 (26.3\%) of 38 cases. Among 20 cases with significant CIPN during weekly paclitaxel, 10 (50\%) cases showed persistent CIPN. Meanwhile, no persistent CIPN was observed in cases without significant CIPN during weekly paclitaxel. These findings suggest that prevention of CIPN during taxane-based therapy is associated with a reduction in the occurrence of persistent CIPN.

Previous clinical trials showed the possibility of cryotherapy or compression therapy to decrease incidence of CIPN during taxane-based chemotherapy in breast cancer patients $[1,2,4,14,15,18]$. In 
addition to these findings, our study also showed the possibility of cryotherapy in prevention of persistent CIPN after completion of weekly paclitaxel. Although there was no significant difference between the cryotherapy and control groups due to the nature of unplanned analysis, less than half of cases in this study experienced persistent CIPN in the cryotherapy group compared with the control group $(15.8 \% \mathrm{vs}$. $36.8 \%$ ). PNQ also showed lower grade persistent CIPN in the cryotherapy group. Since the incidence of CIPN during chemotherapy is a risk factor for persistent CIPN, it is logical that prevention of CIPN during chemotherapy leads to the reduction of persistent CIPN.

As with previous study, age is an important factor for the incidence of persistent CIPN. In a randomized trial of Acetyl-L-Carnitine for prevention of taxane-induced CIPN, women aged 60 years or older had an increased risk of CIPN compared with women younger than age 60 years at a 1 and 2 year follow-up[7]. Our study showed that age 60 or more was a significant predictive factor for persistent CIPN. The incidence of persistent CIPN was $8.7 \%$ and $53.3 \%$ in the age $<60$ and age 60 or older, respectively. The precise mechanism is unknown, however, aging is at least a risk factor for CIPN in patients treated with paclitaxel. A cohort study including 333 participants to identify the association of clinical factors with CIPN persistence in patients treated with paclitaxel or oxaliplatin showed that an older age was associated with worse CIPN in multivariate analysis[11]. A cohort study of the Southwest Oncology Group database that evaluated the incidence of CIPN in clinical trials showed that the incidence of grade2-4 taxane-induced CIPN increases by $4 \%$ for each year of age[6]. In this study, the incidence of persistent CIPN was $33.3 \%$ and $66.7 \%$ in the cryotherapy group and control group, respectively, in age 60 or older. In comparison, there was no difference ( $7.7 \%$ vs. $10 \%)$ between these groups at an age less than 60 . These findings suggest that pronounced precaution should be taken to prevent CIPN when older patients are treated with weekly paclitaxel.

Our study has several limitations. First, this is an unplanned study of cases enrolled in a previous clinical trial and there was a lack of statistical power to detect the difference between cryotherapy and the control group. Second, evaluation of persistent CIPN in this study was based on a subjective patient questionnaire. Objective evaluation, such as the Semmes-Weinstein monofilament test, should be incorporated to improve the robustness of our finding. A prospective randomized trial with a long-term period is warranted to confirm the preventive effect of cryotherapy on persistent CIPN $[9,10]$.

\section{Declarations}

Funding: This study received no funding.

Conflicts of interest/Competing interests: The authors declare that they have no conflict of interest.

Availability of data and material: Data and material are available

Code availability: Not applicable. 
Authors' contributions: HS: study concept, design, data analysis, and writing of the manuscript. YK, TI, and DY: study concept and design. All authors reviewed final manuscript.

Ethics approval: This study was approved by the Ethics Committee of the National Hospital Organization of Kure Medical Center and Chugoku Cancer Center (Approval number 28-70) and adhered to the Helsinki Declaration and the ethical principles for clinical research.

Consent to participate: All patients received a detailed explanation of the study from the primary physician and informed consent was obtained prior to enrollment.

Consent for publication: Informed consent to have de-identified data published was obtained from all individual participants included in the study.

\section{Acknowledgements}

We thank Research Associate (Lecturer) Tomoyuki Akita of Department of Epidemiology, Infectious Disease Control and Prevention, Graduate School of Biomedical and Health Sciences, Hiroshima University (Hiroshima, Japan) for critically reviewing the statistical analysis of this study.

\section{Ethical approval}

This study was approved by the Ethics Committee of the National Hospital Organization of Kure Medical Center and Chugoku Cancer Center (Approval number 28-70) and adhered to the Helsinki Declaration and the ethical principles for clinical research. All patients received a detailed explanation of the study from the primary physician and informed consent was obtained prior to enrollment.

\section{References}

1. Bandla A, Sundar R, Liao LD, Sze Hui Tan S, Lee SC, Thakor NV, Wilder-Smith EP (2016) Hypothermia for preventing chemotherapy-induced neuropathy - a pilot study on safety and tolerability in healthy controls. Acta oncologica 55:430-436

2. Bandla A, Tan S, Kumarakulasinghe NB, Huang Y, Ang S, Magarajah G, Hairom Z, Lim JSJ, Wong A, Chan G, Ngoi N, Ang E, Lee YM, Chan A, Lee SC, Thakor N, Wilder-Smith E, Sundar R (2020) Safety and tolerability of cryocompression as a method of enhanced limb hypothermia to reduce taxaneinduced peripheral neuropathy Supportive care in cancer:. official journal of the Multinational Association of Supportive Care in Cancer 28:3691-3699

3. Cella D, Peterman A, Hudgens S, Webster K, Socinski MA (2003) Measuring the side effects of taxane therapy in oncology: the functional assesment of cancer therapy-taxane (FACT-taxane). Cancer 98:822-831

4. Hanai A, Ishiguro $H$, Sozu T, Tsuda $M$, Yano I, Nakagawa T, Imai S, Hamabe $Y$, Toi M, Arai $H$, Tsuboyama T (2018) Effects of Cryotherapy on Objective and Subjective Symptoms of PaclitaxelInduced Neuropathy: Prospective Self-Controlled Trial. J Natl Cancer Inst 110:141-148 
5. Hershman DL, Lacchetti C, Dworkin RH, Lavoie Smith EM, Bleeker J, Cavaletti G, Chauhan C, Gavin P, Lavino A, Lustberg MB, Paice J, Schneider B, Smith ML, Smith T, Terstriep S, Wagner-Johnston N, Bak K, Loprinzi CL, American Society of Clinical O (2014) Prevention and management of chemotherapyinduced peripheral neuropathy in survivors of adult cancers: American Society of Clinical Oncology clinical practice guideline. Journal of clinical oncology: official journal of the American Society of Clinical Oncology 32:1941-1967

6. Hershman DL, Till C, Wright JD, Awad D, Ramsey SD, Barlow WE, Minasian LM, Unger J (2016) Comorbidities and Risk of Chemotherapy-Induced Peripheral Neuropathy Among Participants 65 Years or Older in Southwest Oncology Group Clinical Trials. Journal of clinical oncology: official journal of the American Society of Clinical Oncology 34:3014-3022

7. Hershman DL, Unger JM, Crew KD, Till C, Greenlee H, Minasian LM, Moinpour CM, Lew DL, Fehrenbacher L, Wade JL 3rd, Wong SF, Fisch MJ, Lynn Henry N, Albain KS (2018) Two-Year Trends of Taxane-Induced Neuropathy in Women Enrolled in a Randomized Trial of Acetyl-L-Carnitine (SWOG S0715. J Natl Cancer Inst 110:669-676

8. Hershman DL, Weimer LH, Wang A, Kranwinkel G, Brafman L, Fuentes D, Awad D, Crew KD (2011) Association between patient reported outcomes and quantitative sensory tests for measuring longterm neurotoxicity in breast cancer survivors treated with adjuvant paclitaxel chemotherapy. Breast cancer research treatment 125:767-774

9. Jordan B, Margulies A, Cardoso F, Cavaletti G, Haugnes HS, Jahn P, Le Rhun E, Preusser M, Scotte F, Taphoorn MJB, Jordan K, clinicalguidelines@esmo.org EGCEa, eons.secretariat@cancernurse.eu EEWGEa, office@eano.eu EGCEa (2020) Systemic anticancer therapy-induced peripheral and central neurotoxicity: ESMO-EONS-EANO Clinical Practice Guidelines for diagnosis, prevention, treatment and follow-up Annals of oncology: official journal of the European Society for Medical Oncology 31: $1306-1319$

10. Loprinzi CL, Lacchetti C, Bleeker J, Cavaletti G, Chauhan C, Hertz DL, Kelley MR, Lavino A, Lustberg MB, Paice JA, Schneider BP, Lavoie Smith EM, Smith ML, Smith TJ, Wagner-Johnston N, Hershman DL (2020) Prevention and Management of Chemotherapy-Induced Peripheral Neuropathy in Survivors of Adult Cancers: ASCO Guideline Update. Journal of clinical oncology: official journal of the American Society of Clinical Oncology 38:3325-3348

11. Mizrahi D, Park SB, Li T, Timmins HC, Trinh T, Au K, Battaglini E, Wyld D, Henderson RD, Grimison P, Ke H, Geelan-Small P, Marker J, Wall B, Goldstein D (2021) Hemoglobin, Body Mass Index, and Age as Risk Factors for Paclitaxel- and Oxaliplatin-Induced Peripheral Neuropathy. JAMA network open 4:e2036695

12. Mols F, Beijers T, Vreugdenhil G, van de Poll-Franse L (2014) Chemotherapy-induced peripheral neuropathy and its association with quality of life: a systematic review Supportive care in cancer:. official journal of the Multinational Association of Supportive Care in Cancer 22:2261-2269

13. Mustafa Ali M, Moeller M, Rybicki L, Moore HCF (2017) Long-term peripheral neuropathy symptoms in breast cancer survivors. Breast cancer research treatment 166:519-526 
14. Rosenbaek F, Holm HS, Hjelmborg JVB, Ewertz M, Jensen JD (2020) Effect of cryotherapy on dose of adjuvant paclitaxel in early-stage breast cancer Supportive care in cancer:. official journal of the Multinational Association of Supportive Care in Cancer 28:3763-3769

15. Shigematsu H, Hirata T, Nishina M, Yasui D, Ozaki S (2020) Cryotherapy for the prevention of weekly paclitaxel-induced peripheral adverse events in breast cancer patients Supportive care in cancer:. official journal of the Multinational Association of Supportive Care in Cancer 28:5005-5011

16. Shimozuma K, Ohashi Y, Takeuchi A, Aranishi T, Morita S, Kuroi K, Ohsumi S, Makino H, Mukai H, Katsumata N, Sunada Y, Watanabe T, Hausheer FH (2009) Feasibility and validity of the Patient Neurotoxicity Questionnaire during taxane chemotherapy in a phase III randomized trial in patients with breast cancer: N-SAS BC 02 Supportive care in cancer. official journal of the Multinational Association of Supportive Care in Cancer 17:1483-1491

17. Tolaney SM, Guo H, Pernas S, Barry WT, Dillon DA, Ritterhouse L, Schneider BP, Shen F, Fuhrman K, Baltay M, Dang CT, Yardley DA, Moy B, Marcom PK, Albain KS, Rugo HS, Ellis MJ, Shapira I, Wolff AC, Carey LA, Overmoyer B, Partridge AH, Hudis CA, Krop IE, Burstein HJ, Winer EP (2019) Seven-Year Follow-Up Analysis of Adjuvant Paclitaxel and Trastuzumab Trial for Node-Negative, Human Epidermal Growth Factor Receptor 2-Positive Breast Cancer. Journal of clinical oncology: official journal of the American Society of Clinical Oncology 37:1868-1875

18. Tsuyuki S, Senda N, Kanng Y, Yamaguchi A, Yoshibayashi H, Kikawa Y, Katakami N, Kato H, Hashimoto T, Okuno T, Yamauchi A, Inamoto T (2016) Evaluation of the effect of compression therapy using surgical gloves on nanoparticle albumin-bound paclitaxel-induced peripheral neuropathy: a phase II multicenter study by the Kamigata Breast Cancer Study Group Breast cancer research and treatment 160: 61-67

\section{Tables}

Table 1. clinicopathological factors 


\begin{tabular}{|c|c|c|c|c|}
\hline factors & & $\begin{array}{l}\text { cryotherapy } \\
(n=19)\end{array}$ & $\begin{array}{l}\text { Control } \\
(n=19)\end{array}$ & $p$ value $^{*}$ \\
\hline \multirow[t]{2}{*}{ Age, years } & $60>$ & 13 & 10 & 0.3 \\
\hline & $60<=$ & 6 & 9 & \\
\hline Height, mean(SD), cm & & $156.5(5.9)$ & 154.1(7.1) & 0.3 \\
\hline Weight, mean(SD), kg & & $52.3(7.1)$ & $55.9(9.1)$ & 0.2 \\
\hline \multirow[t]{3}{*}{ Stage } & 1 & 4 & 2 & 0.7 \\
\hline & II & 8 & 9 & \\
\hline & III & 7 & 8 & \\
\hline \multirow[t]{2}{*}{ Adjuvant endocrine therapy } & yes & 15 & 16 & 1 \\
\hline & no & 4 & 3 & \\
\hline \multirow[t]{2}{*}{ Adjuvant radiation therapy } & yes & 14 & 15 & 1 \\
\hline & no & 5 & 4 & \\
\hline \multirow[t]{2}{*}{ Decrease in FACT-NTX during weekly paclitaxel ${ }^{\star *}$} & yes & 6 & 14 & 0.008 \\
\hline & no & 13 & 5 & \\
\hline \multirow[t]{5}{*}{ PNQ sensory grade during weekly paclitaxel } & $A$ & 2 & 1 & 0.02 \\
\hline & $\mathrm{B}$ & 11 & 3 & \\
\hline & $\mathrm{C}$ & 5 & 8 & \\
\hline & D & 1 & 6 & \\
\hline & $\mathrm{E}$ & 0 & 1 & \\
\hline \multirow[t]{5}{*}{ PNQ motor grade during weekly paclitaxel } & A & 4 & 0 & 0.04 \\
\hline & B & 11 & 9 & \\
\hline & C & 3 & 6 & \\
\hline & D & 1 & 2 & \\
\hline & $\mathrm{E}$ & 0 & 2 & \\
\hline
\end{tabular}

Abbreviations: SD, standard deviation; FACT-NTX, Functional Assessment of Cancer TherapyNeurotoxicity; PNQ, Patient Neurotoxicity Questionnaire

*Factors were compared by treatment groups using Fisher's exact test for categorical variables and t tests for continuous variables. Tests were two-sided. 
${ }^{* \star}$ A 6-point or more decrease in the total of FACT-NTX score from baseline is regarded as clinically significant CIPN.

Table2. Results of FACT-NTX and PNQ after completion of weekly paclitaxel treatment.

\begin{tabular}{|c|c|c|c|c|}
\hline factors & & $\begin{array}{l}\text { Cryotherapy } \\
(n=19)\end{array}$ & $\begin{array}{l}\text { Control } \\
(n=19)\end{array}$ & $\begin{array}{l}P \\
\text { value }\end{array}$ \\
\hline $\begin{array}{l}\text { Interval from weekly paclitaxel to questionnaire, } \\
\text { years(SD) }\end{array}$ & & $2.5(0.5)$ & $2.6(0.5)$ & 0.4 \\
\hline \multirow[t]{2}{*}{ Significant decrease in FACT-NTX ${ }^{\star *}$} & yes & 3 & 7 & 0.13 \\
\hline & no & 16 & 12 & \\
\hline \multirow[t]{5}{*}{ PNQ sensory grade } & $A$ & 11 & 5 & 0.02 \\
\hline & B & 6 & 8 & \\
\hline & C & 0 & 5 & \\
\hline & D & 2 & 1 & \\
\hline & E & 0 & 0 & \\
\hline \multirow[t]{5}{*}{ PNQ motor grade } & A & 14 & 11 & 0.17 \\
\hline & B & 3 & 4 & \\
\hline & C & 0 & 3 & \\
\hline & D & 2 & 1 & \\
\hline & E & 0 & 0 & \\
\hline
\end{tabular}

Abbreviations: SD, standard deviation; FACT-NTX, Functional Assessment of Cancer TherapyNeurotoxicity; PNQ, Patient Neurotoxicity Questionnaire

*Factors were compared by treatment groups using Fisher's exact test. Tests were two sided.

${ }^{\star \star} \mathrm{A}$ 6-point or more decrease in the total of FACT-NTX score from baseline is regarded as clinically significant CIPN.

\section{Figures}




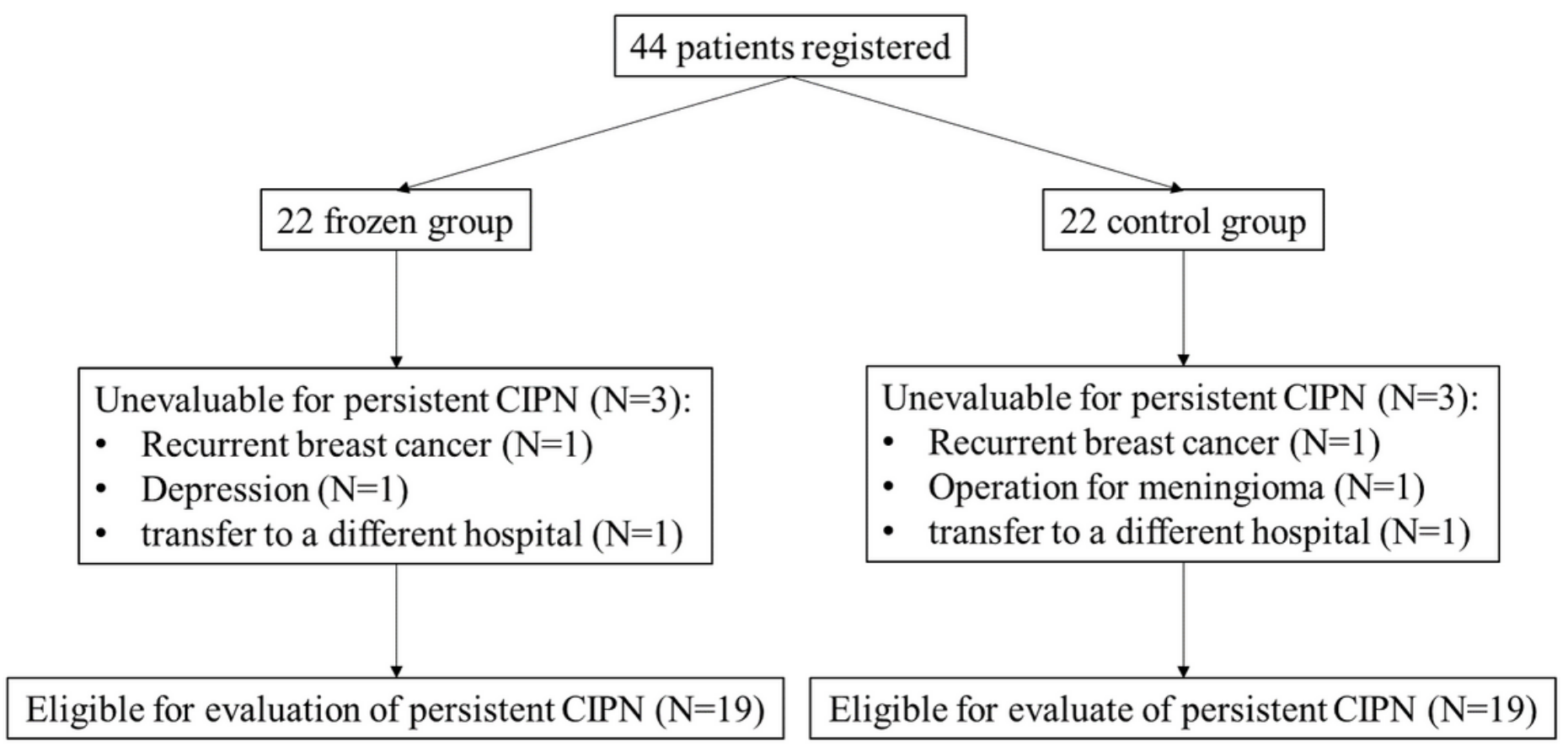

Figure 1

Consort diagram for this survey

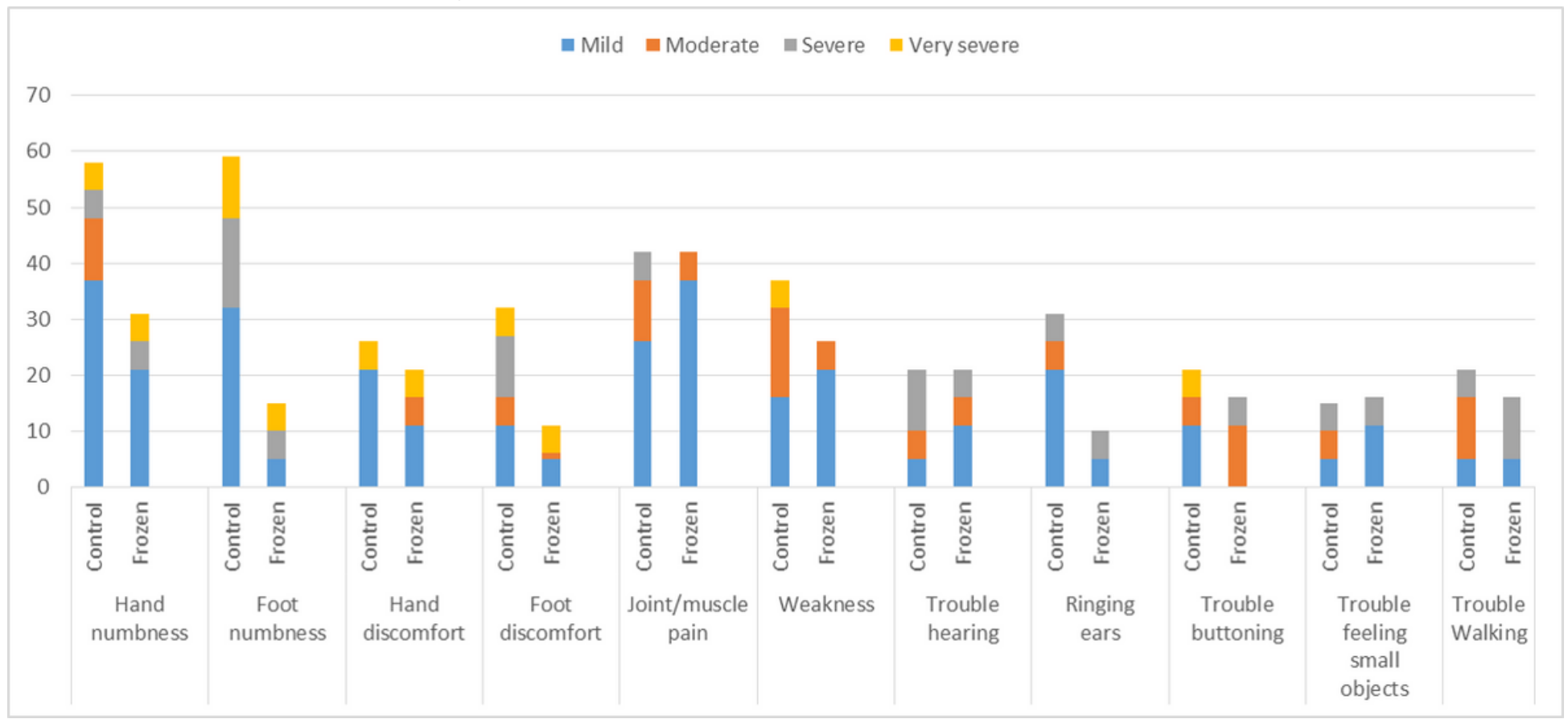

Figure 2

Distribution of symptom grade for each questionnaire item of FACT-NTX after weekly paclitaxel in cryotherapy and control group. 